\title{
Surface Waves on Self-Complementary Metasurfaces: All-Frequency Hyperbolicity, Extreme Canalization, and TE-TM Polarization Degeneracy
}

\author{
Oleh Yermakov $\odot,{ }^{1, *}$ Vladimir Lenets, ${ }^{1, *}$ Andrey Sayanskiy $\odot,{ }^{1}$ Juan Baena $\odot,{ }^{2}$ \\ Enrica Martini $\odot,{ }^{3}$ Stanislav Glybovski $\odot,{ }^{1, \dagger}$ and Stefano Maci ${ }^{3}$ \\ ${ }^{1}$ School of Physics and Engineering, ITMO University, St. Petersburg 197101, Russia \\ ${ }^{2}$ Department of Physics, Universidad Nacional de Colombia, Bogota 111321, Colombia \\ ${ }^{3}$ Department of Information Engineering and Mathematics, University of Siena, Siena 53100, Italy
}

(Received 14 April 2020; revised 16 May 2021; accepted 15 June 2021; published 18 August 2021)

\begin{abstract}
Self-complementary metasurfaces have gained significant attention due to their unique frequencyindependent transmission and reflection properties and the possibility of the polarization transformation of plane waves. In this paper, we focus on the near-field spectrum to investigate, both theoretically and experimentally, the properties of surface waves supported by anisotropic self-complementary metasurfaces. We show that as a consequence of the electromagnetic Babinet's duality, such a structure is hyperbolic for any frequency. We demonstrate the possibility of switching the canalization direction of surface waves with ultimately flat phase fronts for $90^{\circ}$ by a very small frequency shift, paving the way to the extreme tunability and surface-wave routing. We reveal the polarization degree of freedom inherent to plane waves by demonstrating the all-frequency TE-TM polarization degeneracy of the surface waves along two principal directions. The results obtained open a plethora of opportunities for practical applications, including flat polarization devices, optical data-processing systems, sensing, holography and antennas.
\end{abstract}

DOI: 10.1103/PhysRevX.11.031038

Subject Areas: Metamaterials, Photonics, Plasmonics

\section{INTRODUCTION}

Metasurfaces have gained significant attention because of their ability to control the phase, amplitude, and polarization of the incident electromagnetic waves in transmission and reflection [1-3]. Metasurfaces represent a thin-layer periodic array of subwavelength scatterers tailored to achieve the necessary goals in electromagnetic-waves control for a number of practical applications such as lenses, antennas, absorbers, filters, polarizers, holograms, etc., [4,5]. An even more promising fact is that metasurfaces provide the ultrathin platform to implement on-chip and photonic devices based on nearfield effects and in-plane propagation of surface plasmonpolaritons with a plethora of perspective applications in communications, sensing, and optical networks [6,7]. Another important application area is the microwave and millimeter-wave antennas, where surface waves propagating at spatially nonuniform impedance metasurfaces have

\footnotetext{
*These authors contributed equally to this work.

†s.glybovski@metalab.ifmo.ru
}

Published by the American Physical Society under the terms of the Creative Commons Attribution 4.0 International license. Further distribution of this work must maintain attribution to the author(s) and the published article's title, journal citation, and DOI. been used. Antennas can be realized by converting slow surface waves into radiating waves by introducing a periodic or quasiperiodic spatial modulation of the surface impedance [8]. This method was successfully employed to design low-profile and high-gain directive antennas with radiation pattern shapes precisely controlled by the impedance modulation [9-11]. Furthermore, Luneburg [12-14] and Maxwell fisheye [15] flat microwave lenses were based on engineered curvilinear propagation of surface waves over a spatially nonuniform metasurface.

Anisotropic self-complementary metasurfaces are singlelayer metal patterns that remain the same after Babinet inversion except for some translation or rotation. They were shown to operate as frequency-selective filters [16], perfect absorbers [17], and linear-to-circular polarization converters for incident plane waves $[18,19]$, and they have frequencyconstant transmission properties when excited by circularly polarized waves [20]. In contrast to isotropic checkerboard, which has self-complementary patterns requiring perfectly sharp corners of planar square conductors [21-23], their anisotropic counterparts are constructed of alternating inductive and capacitive complementary strips and, therefore, are easy to implement. In Refs. [24,25], it was shown that two mutually complementary surfaces, one with inductive and the other with capacitive impedance, have the same dispersion of TM and TE surface waves, respectively. However, surface waves on anisotropic self-complementary metasurfaces have not previously been studied. 
In this work, we study, in detail, the fundamental properties of surface waves on self-complementary metasurfaces. We consider a symmetric case of a nonchiral metasurface exhibiting no cross-polarization response in an appropriate Cartesian basis and, as a consequence, with a diagonalized effective local surface admittance tensor $\hat{Y}_{0}=\operatorname{diag}\left(Y_{x}, Y_{y}\right)$. Then, we provide the self-consistent analytical model supported by numerical and experimental results. The self-complementary metasurface is defined as one obeying the Babinet's duality relation:

$$
\operatorname{det} \hat{Y}_{0}=Y_{x} Y_{y}=4 Y_{0}^{2},
$$

where $Y_{0}=1 / Z_{0}$ and $Z_{0}$ is the free space wave impedance $[22,26]$. In addition to the fundamental investigation of self-complementary metasurfaces, we raise three topical issues related to the near-field control over surface waves and demonstrate the benefits of self-complementary metasurfaces over general anisotropic ones.

Overcoming frequency limitation for hyperbolic plasmon-polaritons.-We pay particular attention to hyperbolic plasmons-surface waves localized at hyperbolic metasurfaces [27-30]. For the last decade, many exciting regimes of hyperbolic plasmon propagation were discovered and analyzed, including negative refraction [31], zero index [32], Moiré patterns [33,34], unidirectional [35,36], and canalization [37] regimes of propagation. Despite the great success in hyperbolic plasmon research, the potential for further development and application is strictly limited by the narrow operational frequency range corresponding to the hyperbolicity condition $\operatorname{Im}\left(Y_{x}\right) \operatorname{Im}\left(Y_{y}\right)<$ 0 [Figs. 1(a) and 1(b)].

Canalization of surface waves: Propagation with ultimately flat phase fronts and direction switching.-The canalization [39] regime is a well-known phenomenon in photonic crystals [40,41], wire medium [42], and epsilonnear-zero metamaterials [43,44] exhibiting flat isofrequency contours in the reciprocal wave-vector space $\left(k_{x}, k_{y}\right)$. It leads to the enhanced resolution (greater than diffraction limit), imaging, and lensing [45-47]. Recently, the study of the surface-wave canalization regime at the anisotropic metasurface has gained great

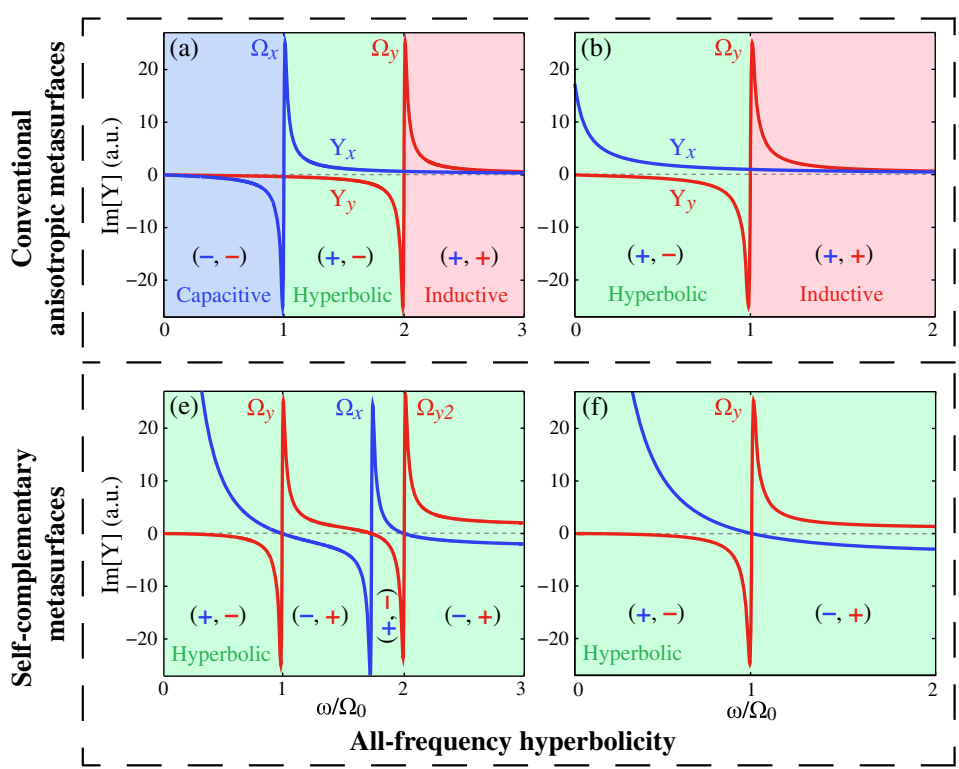

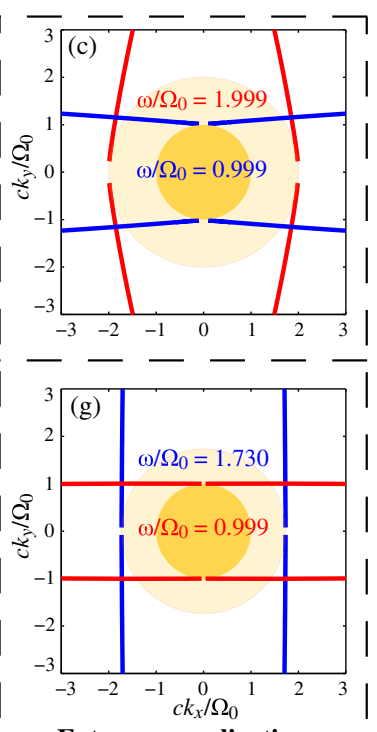

Extreme canalization

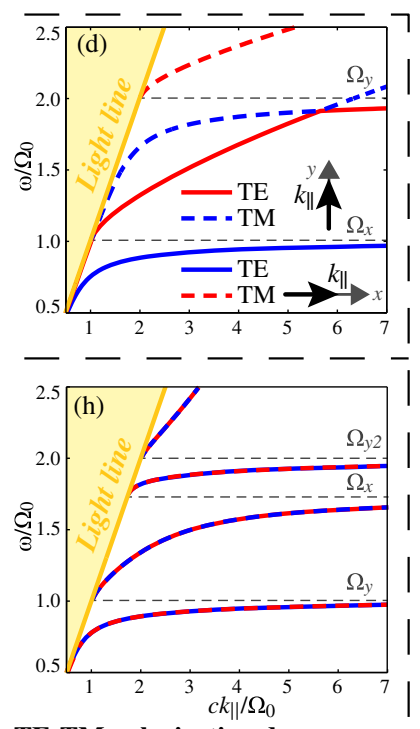

TE-TM polarization degeneracy

FIG. 1. Three topical issues related to the near-field properties of surface waves on the conventional anisotropic two-dimensional systems (a)-(d) and self-complementary metasurfaces (e)-(h). (a,b,e,f) I. Hyperbolic regime of a metasurface corresponding to $\operatorname{Im}\left(Y_{x}\right) \operatorname{Im}\left(Y_{y}\right)<0$. The dependence of the imaginary parts of the admittance tensor components $Y_{x}$ (blue lines) and $Y_{y}$ (red lines) on the frequency in the dimensionless units normalized per the frequency of the first resonance $\Omega_{0}$. The signs "+" and "-_" correspond to the positive and negative values of $\left(Y_{x}, Y_{y}\right)$, respectively. The green area corresponds to the hyperbolic regime of a metasurface. The resonant frequencies of the conventional anisotropic $2 \mathrm{D}$ systems $\left(\Omega_{x}, \Omega_{y}\right)$ and the self-complementary metasurfaces $\left(\Omega_{y}, \Omega_{y 2}\right)$ are spectrally (a,e) close [namely, (a) $\Omega_{y} / \Omega_{x}=2$, (e) $\Omega_{y 2} / \Omega_{y}=2$ ] and (b,f) far from each other [namely, (b) $\Omega_{x} / \Omega_{y} \ll 1$, (f) $\left.\Omega_{y 2} / \Omega_{y} \ll 1\right]$. $(\mathrm{c}, \mathrm{g})$ II. Extreme (divergenceless) canalization corresponding to absolutely flat isofrequency contours. (c,g) Isofrequency contours in the vicinity of the resonances (c) $\Omega_{x}$ (blue line) and $\Omega_{y}$ (red line) for conventional anisotropic metasurface, and (g) $\Omega_{y}$ (blue line) and $\Omega_{x}$ (red line) for the self-complementary metasurface. The circles correspond to the light cone at the same frequencies. (d,h) III. All-frequency TE-TM polarization degeneracy. We show the dependence of frequency on the in-plane wave-vector component $k_{\|}$for TE- and TMpolarized surface waves propagating along the $x$ and $y$ directions for (d) conventional anisotropic and (h) self-complementary metasurfaces. The yellow region corresponds to the light cone. The parameters of the surface admittance tensor components for hyperbolic and self-complementary metasurfaces can be found in the Supplemental Material [38]. 
importance because of the enormous application potential in flat devices, on-chip networks, and optical signal control $[6,37,48]$. This phenomenon is observed in the extremely anisotropic systems in the vicinity of the nearzero regimes. This exciting effect is still poorly used and remains exotic in the aspect of applications owing to the nontunable single-frequency and unidirectional operational regime that typically exhibits a weak divergence [Fig. 1(c)] that is perceptible for the long-range applications.

Polarization degree of freedom for surface waves.Plane waves in any isotropic medium are double degenerate with respect to polarization in all directions at any frequency. The surface waves at anisotropic metasurfaces possess hybrid TE-TM polarization [49,50], providing an efficient tool for the spin-dependent electromagnetic phenomena [50-54] and polarization-dependent directional plasmon steering [55-59]. However, despite the complicated polarization structure of surface waves, the development of flat optics is significantly limited by the inefficient polarization control of the propagating localized electromagnetic waves. Except for accidental intersections at several points, the dispersion curves of TE and TM surface modes are not degenerate [Fig. 1(d)], in sharp contrast to the plane waves in an isotropic medium. Nevertheless, polarization degeneracy of the TE and TM modes is the fundamental operational principle of the classical bulk polarizers. The polarization transformation of propagating surface waves also requires the broadband TE-TM polarization degeneracy of the eigenmode spectrum inherent to the bulk waves.

In this work, we show that, thanks to the selfcomplementariness, the above-mentioned benefits are achievable. In particular, self-complementary metasurfaces exhibit (1) all-frequency hyperbolicity as illustrated in Figs. 1(e) and 1(f), (2) the divergenceless and dualdirectional canalization of surface waves switched by a very small frequency shift [Fig. 1(g)], and (3) the allfrequency TE-TM polarization degeneracy of surface waves [Fig. 1(h)]. Particularly, we show the canalization of polarization-degenerate surface waves leading to the efficient signal routing preserving the polarization of its source, as schematically depicted in Fig. 2.

The present paper is organized as follows. Section II presents the developed analytical model describing the properties of surface waves on self-complementary metasurfaces within an effective surface admittance approach. In Sec. III, we provide the numerically optimized design of a metasurface for the practical implementation, describe the experimental setup and measurement methods, and compare the analytical, numerical, and experimental results validating their relevance. In Sec. IV we study, in detail, the main results and features of self-complementary metasurfaces by dividing them into the three topical issues

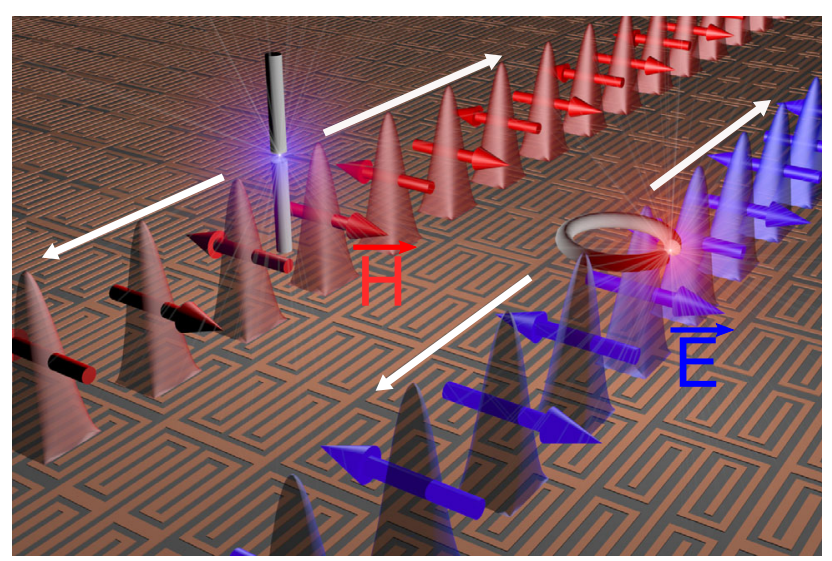

FIG. 2. Artistic representation of the canalized and polarization-degenerate surface waves at the self-complementary metasurface under study. The red and blue arrows demonstrate the instantaneous direction of magnetic and electric fields of TM and TE surface plasmons excited by vertical electric (probe) and magnetic (loop) dipolelike sources, respectively. The conical shapes schematically show the field amplitude sharply decreasing with distance from a metasurface. The white arrows correspond to the wave propagation directions emulating the canalization propagation regime of the surface plasmon-polaritons.

mentioned above. Finally, concluding remarks and an outlook are given in Sec. V.

\section{FORMULATION}

\section{A. Problem statement}

We consider a freestanding single-layer resonant anisotropic metasurface constituted by alternance of infinitely thin complementary inductive and capacitive strips with narrow width in terms of the operating wavelength (Fig. 3); thus, they can be homogenized with boundary condition

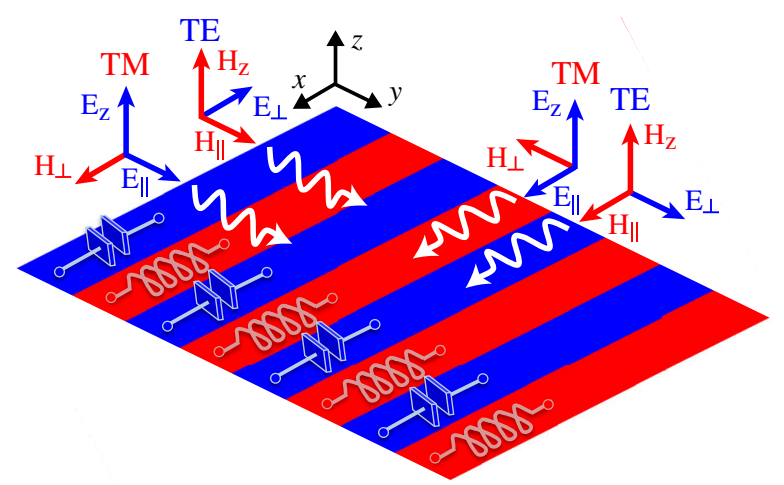

FIG. 3. Geometry of the problem constituted by a collections of coplanar inductive and capacitive parallel strips of a width that is small in terms of a wavelength immersed in free space. The polarization of TE and TM surface-wave modes with a propagation direction both parallel and orthogonal to the strips is also depicted. 


$$
\mathbf{J}=\hat{\mathbf{n}} \times\left(\mathbf{H}_{\mathbf{1}}-\mathbf{H}_{\mathbf{2}}\right)=\hat{Y} \mathbf{E}_{\tau} .
$$

In Eq. (2), $\hat{\mathbf{n}}$ is the unit normal vector from the lower to the upper half-space, $\mathbf{J}$ is a surface electric current density proportional to the jump of the tangential component of the magnetic field across the metasurface plane, $\mathbf{E}_{\tau}$ is a tangential electric field component that is continuous across the metasurface, and $\hat{Y}$ is the local effective surface admittance tensor describing the anisotropic metasurface.

It is worth noting that the above structure respects the Babinet's duality principle if the strips are mutually complementary. This result can be obtained by realizing geometrical complementary slots and dipoles along the strips while still keeping them compact in terms of a wavelength. If two half-spaces above and below are filled with different permittivities, the structure, strictly speaking, does not respect the duality. Nevertheless, the physical mechanism we describe will be the same. First, we analyze an ideally dual case, while in the experiment, we implement a metasurface as a self-complementary copper pattern on a thin dielectric substrate. As we will show, in the considered practical metasurface realization, the Babinet's duality principle is still fulfilled well.

\section{B. Admittance tensor}

The anisotropic metasurfaces, as well as other ultrathin subwavelength systems (graphene, van der Waals materials, 2D electron gas, etc.) can be reasonably described in terms of the effective surface admittance (conductivity) approach. We assume that a metasurface creates no crosspolarization when the incident plane wave has only an $x$ - or a $y$-directed tangential electric field. Thus, in these coordinates, the tensor can be diagonalized along the principal axes as follows:

$$
\hat{Y}_{0}=\left(\begin{array}{cc}
Y_{x} & 0 \\
0 & Y_{y}
\end{array}\right) .
$$

The resonant response of a metasurface can be expressed by a Lorentzian form of one of the admittance tensor components (for example, here we have chosen the $Y_{y}$ component):

$$
Y_{y}=\sum_{j} \frac{i Y_{0} N_{j} \omega^{2}}{\omega^{2}-\Omega_{j}^{2}+i \gamma_{j} \omega} .
$$

Here, $j$ denotes the resonance number, $N_{j}$ is a nondimensional normalization factor, $\Omega_{j}$ is the resonance angular frequency, and $\gamma_{j}$ is the resonance bandwidth.

According to the Babinet's duality relation, the surface admittance tensor components obey the relation (1). Thus, the opposite admittance tensor component $Y_{x}$ can be strictly determined by the relation (1) and is inversely proportional to the one defined in Eq. (4). We note that the duality relation (1) highlights a cancellation between the poles and the zeros of the admittances [Fig. 4(a)], which implies a frequency alternance between series and paralleltype resonances for the two eigenvalues of the tensor.

In order to find the expression of the admittance in an arbitrary reference system, it is useful to introduce a wavevector-fixed coordinate system constituted by unit vectors parallel and orthogonal to the direction of propagation. Assuming that the wave vector forms an angle $\alpha$ with respect to the $x$ axis, in this reference system, the admittance tensor may be written as

$$
\hat{Y}=\left(\begin{array}{cc}
Y_{\|,\|} & Y_{\|, \perp} \\
Y_{\perp, \|} & Y_{\perp, \perp}
\end{array}\right),
$$

where

$$
\begin{aligned}
Y_{\|,\|} & =Y_{x} \cos ^{2} \alpha+Y_{y} \sin ^{2} \alpha, \\
Y_{\perp, \perp} & =Y_{x} \sin ^{2} \alpha+Y_{y} \cos ^{2} \alpha, \\
Y_{\|, \perp} & =Y_{\perp, \|}=\left(Y_{x}-Y_{y}\right) \cos \alpha \sin \alpha .
\end{aligned}
$$

When $\alpha$ is zero, Eq. (6) recovers Eq. (3); when it is $90^{\circ}$, the tensor again becomes diagonal with a swapped position of the diagonal eigenvalues with respect to $\alpha=0$. In this system (see Fig. 3), the resonances change roles with respect to propagation along the strips; namely, the parallel resonances become series resonances and vice versa, as mentioned before.

\section{Dispersion equation}

The dispersion equation of surface waves at an anisotropic metasurface described with the effective local surface admittance tensor can be found analytically [27]:

$$
\left(\frac{\varepsilon_{1} k_{0}}{\kappa_{1}}+\frac{\varepsilon_{2} k_{0}}{\kappa_{2}}+i \tilde{Y}_{\|,\|}\right)\left(\frac{\kappa_{1}}{k_{0}}+\frac{\kappa_{2}}{k_{0}}-i \tilde{Y}_{\perp, \perp}\right)=\tilde{Y}_{\|, \perp} \tilde{Y}_{\perp, \|} .
$$

Here, $k_{0}=\omega / c, \quad \kappa_{1,2}=\sqrt{k_{\|}^{2}-\varepsilon_{1,2} k_{0}^{2}}$ is a penetration depth, $k_{\|}$is the surface-wave propagation constant, and $\varepsilon_{1}$ and $\varepsilon_{2}$ are the dielectric constants for $z$ positive and negative, respectively. In Eq. (7) and after, we use the following admittance normalization: $\tilde{Y}=Y / Y_{0}$.

One can see that the first and second factors in the left side of Eq. (7) correspond to the dispersion laws of TM and TE modes, respectively, while the right side of Eq. (7) contains a coupling factor related to anisotropy. So, the spectrum of an anisotropic metasurface consists of the modes with hybrid TE-TM polarization, usually called quasi-TM and quasi-TE [27,49] depending on which polarization component is dominant. Importantly, the surface modes possess purely orthogonal TE and TM polarizations in the main axes directions $\alpha=n \pi / 2$, where $n$ is 

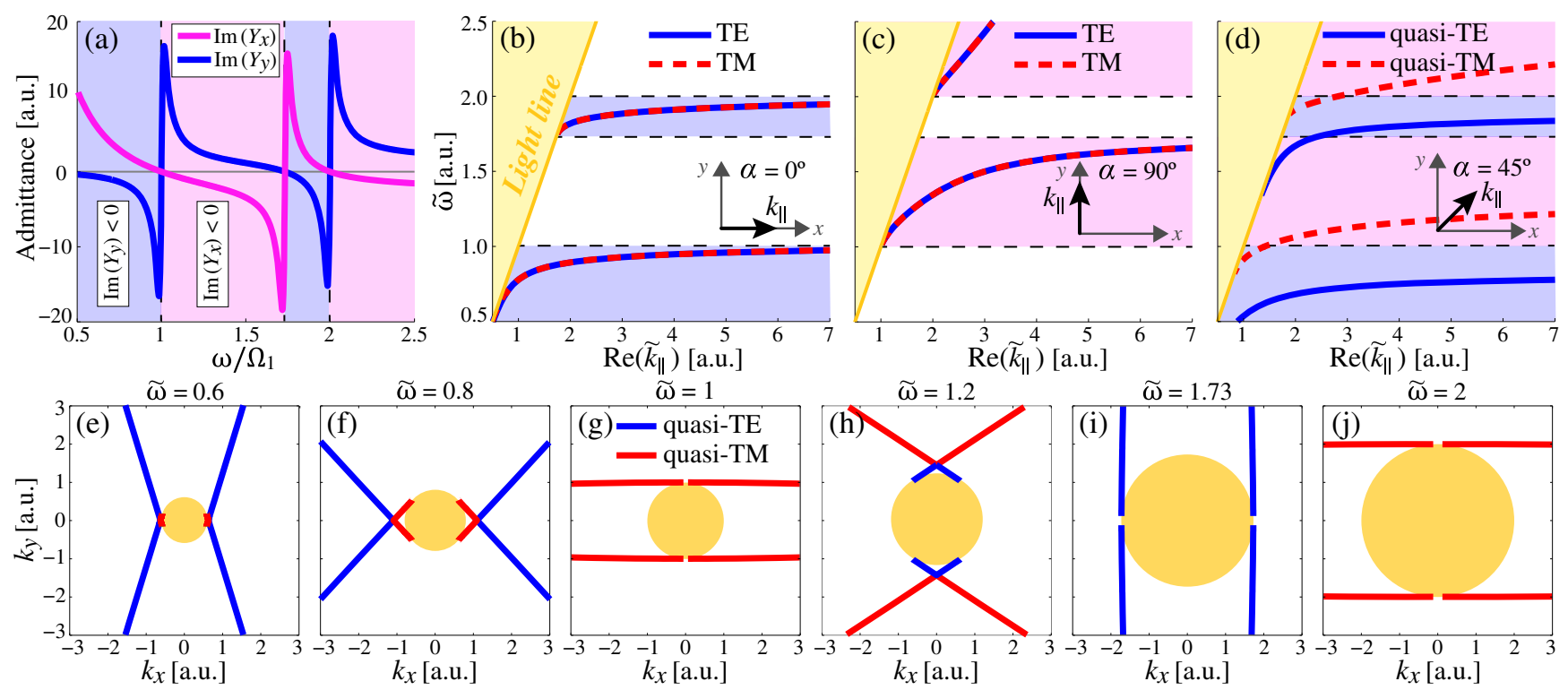

FIG. 4. Analytically calculated properties of an ideal resonant self-complementary metasurface. (a) Frequency dependence of the imaginary parts of admittance tensor components. The Lorentzian parameters are as follows: $N_{1}=1, \tilde{\Omega}_{1}=1, N_{2}=0.5, \tilde{\Omega}_{2}=2$, $\tilde{\gamma}_{1}=\tilde{\gamma}_{2}=0.03$. (b)-(d) Dispersion of surface waves localized at a self-complementary metasurface in different directions $\alpha=0^{\circ}$ (b), $90^{\circ}$ (c), and $45^{\circ}$ (d). (e)-(j) Isofrequency contours of quasi-TE and quasi-TM surface modes in $\left(k_{x}=\tilde{k}_{\|} \cos \alpha, k_{y}=\tilde{k}_{\|}\right.$sin $\left.\alpha\right)$ space at the angular frequencies $\tilde{\omega}=0.6$ (e), $\tilde{\omega}=0.8$ (f), $\tilde{\omega}=1(\mathrm{~g}), \tilde{\omega}=1.2(\mathrm{~h}), \tilde{\omega}=1.73$ (i), and $\tilde{\omega}=2$ (j). Here, we use the dimensionless units $\tilde{\Omega}=\Omega / \Omega_{1}, \tilde{\omega}=\omega / \Omega_{1}, \tilde{\gamma}=\gamma / \Omega_{1}$, and $\tilde{k}_{\|}=c k_{0} / \Omega_{1}=\tilde{\omega}$.

an integer. For any propagation angle, different from $\alpha \neq n \pi / 2$, the spectrum represents the set of the hybrid TE-TM surface waves.

The dispersion equation (7) can be solved analytically in the symmetric case $\varepsilon_{1}=\varepsilon_{2}=\varepsilon$ :

$\kappa^{\mathrm{TM}, \mathrm{TE}}=k_{0} \frac{\zeta \pm \sqrt{\zeta^{2}-\varepsilon \tilde{Y}_{\|,\|} \tilde{Y}_{\perp, \perp}}}{-i \tilde{Y}_{\|,\|}}, \quad \zeta=\varepsilon+\frac{\operatorname{det} \hat{\tilde{Y}}_{0}}{4}$

where the square root is defined with a positive real part and the upper (lower) sign corresponds to the TM (TE) mode. In the absence of losses, the term under the square root becomes real and positive with $\zeta=\varepsilon+1$. In free space, we can express the dispersion of the in-plane complex wavevector component of the surface waves localized at the selfcomplementary metasurface by applying the Babinet's duality relation (1) as follows:

$$
k_{\|}=k_{0} \sqrt{1-\left(\frac{2 \pm \sqrt{-\tilde{Y}_{\|, \perp} \tilde{Y}_{\perp, \|}}}{\tilde{Y}_{\|,\|}}\right)^{2}} .
$$

This equation is specialized for the propagation direction along the strips $\left(\alpha=0^{\circ}\right)$, orthogonal to the strips $\left(\alpha=90^{\circ}\right)$, and at $\alpha=45^{\circ}$, respectively, thus leading to

$$
\begin{aligned}
& k_{\|}^{\alpha=0^{\circ}}=\eta_{x} k_{0} \sqrt{1-\frac{\tilde{Y}_{y}^{2}}{4}}=\eta_{x} k_{0} \sqrt{1-\frac{4}{\tilde{Y}_{x}^{2}}}, \\
& k_{\|}^{\alpha=90^{\circ}}=\eta_{y} k_{0} \sqrt{1-\frac{\tilde{Y}_{x}^{2}}{4}}=\eta_{y} k_{0} \sqrt{1-\frac{4}{\tilde{Y}_{y}^{2}}}, \\
& k_{\|}^{\alpha=45^{\circ}}=k_{0} \sqrt{1-\left(\frac{4 \pm \sqrt{-\tilde{Y}_{-}^{2}}}{\tilde{Y}_{+}}\right)^{2}},
\end{aligned}
$$

where $\eta_{x, y}=U\left[\operatorname{Im}\left(Y_{x, y}\right)\right]$ and $U[]$ is the Heaviside unit step function, $\tilde{Y}_{+,-}=\tilde{Y}_{x} \pm \tilde{Y}_{y}$, and the upper (lower) sign for $\alpha=45^{\circ}$ corresponds to quasi-TM (quasi-TE) modes. This relation states that the eigenmodes propagating along the $x$ and $y$ axes exist only within the frequency range corresponding to $\operatorname{Im}\left(Y_{y}\right)<0$ and $\operatorname{Im}\left(Y_{x}\right)<0$, respectively. These propagation constants are plotted in Figs. 4(b)-4(d). We note that in the two principal directions, the degeneracy of the propagation constant for TE and TM waves takes place, while at $45^{\circ}$, the modes exhibit different wave numbers.

It is important to point out that, strictly speaking, any substrate with permittivity different from the permittivity of free space violates the Babinet's duality relation (1). Nevertheless, the dispersion equation (7) remains relevant for any environment, and it could be solved analytically for small differences between superstrate and substrate permittivities using perturbation theory [27]. We further demonstrate, both numerically and experimentally, the 
consistency of the analytically predicted results for the selfcomplementary metasurface at a thin dielectric substrate.

\section{Isofrequency contours}

One of the powerful tools used to analyze the wave propagation features are the isofrequency contours (IFCs). The possible isofrequency contours for the self-complementary metasurface described by the surface admittance tensor, shown in Fig. 4(a), are presented in Figs. 4(e)-4(j). One can follow the evolution from the horizontal [Figs. 4(e) and 4(f)] and vertical [Fig. 4(h)] hyperbolic to the horizontal [Figs. 4(g) and 4(j)] and vertical [Fig. 4(i)] flat IFCs. The hyperbola-like and flat IFCs correspond to the hyperbolic and canalization regimes of a metasurface, respectively. The latter emerges in the vicinity of the resonances. The horizontal flat IFCs are observed in the vicinity of $Y_{y}$ resonances, while $Y_{x}$ is nearly zero; thus, the condition $\left|Y_{y} / Y_{x}\right| \gg 1$ is fulfilled. For vertical, flat IFCs, the opposite situation takes place.

One can see that, for any frequencies except the resonances, the quasi-TE and quasi-TM IFCs have an intersection point along the corresponding main axes, in accordance with Figs. 4(b) and 4(c). Its absence in the nearresonance frequency range corresponding to the flat IFCs is associated with the losses $\left(\gamma_{1}=\gamma_{2}=0.03 \Omega_{1}\right)$.

\section{NUMERICAL ANALYSIS AND MEASUREMENTS}

\section{A. Unit-cell geometry and numerical analysis}

The unit-cell design of the considered metasurface is based on a meandered dipole and a complementary meandered slot (Fig. 5). By numerical simulations, the following geometric parameters were chosen in order to obtain the first resonance of the metasurface at around $5 \mathrm{GHz}$ : periodicity of the square unit cell $A=7 \mathrm{~mm}$, substrate thickness $H_{\text {sub }}=1 \mathrm{~mm}$, and gap and metal strip widths $g_{1}=0.25 \mathrm{~mm}, g_{2}=0.4 \mathrm{~mm}$, $g_{3}=0.2 \mathrm{~mm}$, and $w=0.2 \mathrm{~mm}$. The guidelines for

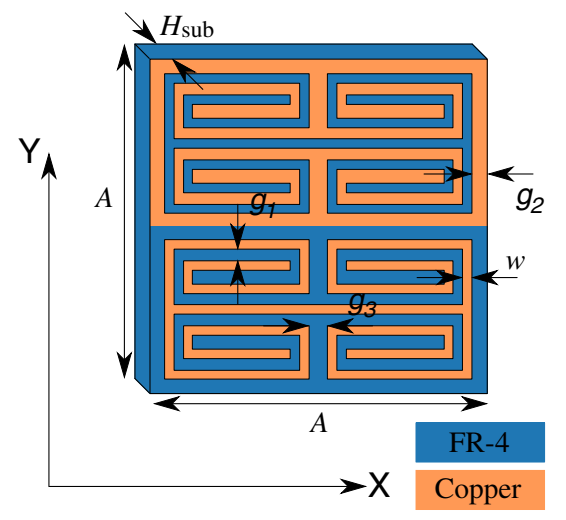

FIG. 5. Unit-cell design of the resonant self-complementary metasurface under investigation.

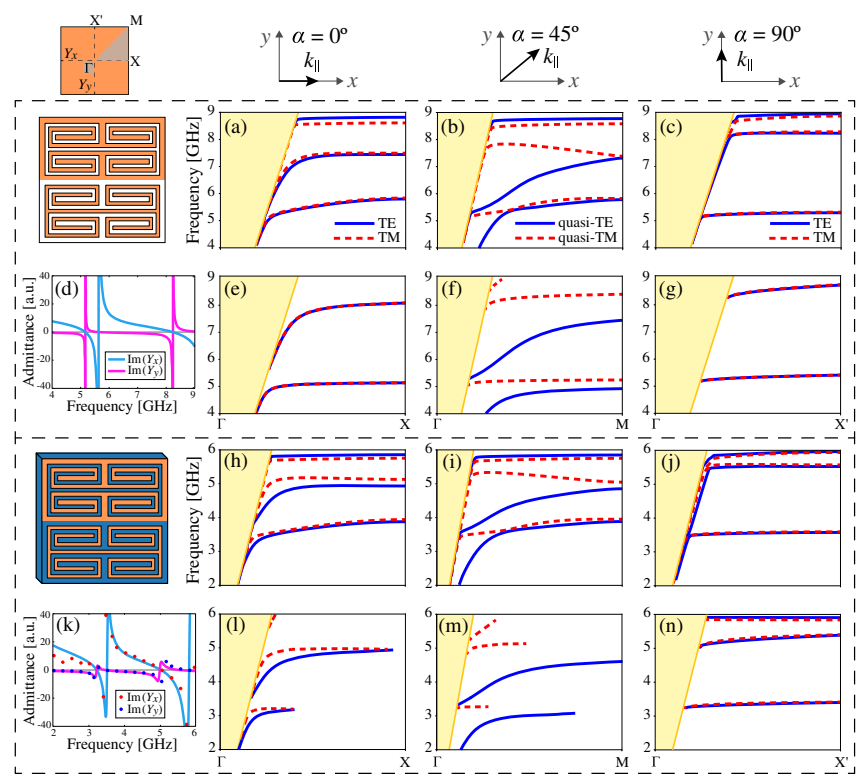

FIG. 6. Dispersion diagrams for surface waves localized at the self-complementary metasurface, shown in Fig. 5, without a substrate (a)-(g) and with a substrate (h)-(n) for different propagation angles $\alpha=0^{\circ}$ (a,e,h,l), $\alpha=45^{\circ}$ (b,f,i,m), and $\alpha=$ $90^{\circ}(\mathrm{c}, \mathrm{g}, \mathrm{j}, \mathrm{n})$. The TE (blue solid line) and TM (red dashed line) dispersion curves were calculated numerically with the CST eigenmode solver [(a)-(c), (h)-(j)] and analytically [Eq. (10)] $[(\mathrm{e})-(\mathrm{g}),(\mathrm{l})-(-\mathrm{n})]$ by using the numerically $(\mathrm{d}, \mathrm{k})$ and experimentally [color dots in $(\mathrm{k})$ ] recovered effective surface admittance tensor.

designing the specific self-complementary metasurface can be found in the Supplemental Material [38].

The numerical simulation of dispersion curves and field patterns over the metasurface, shown in Fig. 5, was done in eigenmodes and transient solvers of CST Microwave Studio, respectively. The surface-wave dispersion comparison between theory, proposed in Sec. II, and numerical simulation is shown in Fig. 6. Semianalytical eigenmode dispersion curves [Figs. 6(e)-6(g), 6(1)-6(n)] are based on the solution of Eq. (7), whereas the effective surface admittance tensor (3) of the real structure (Fig. 5) without [Fig. 6(d)] and with [Fig. 6(k)] the substrate was extracted from the numerically calculated [Figs. 6(d) and 6(k)] and experimentally measured [dots in Fig. 6(k)] S-matrix coefficients [60]. On the other hand, we have numerically found the eigenmode spectrum of the same structure without [Figs. 6(a)-6(c)] and with [Figs. 6(h)-6(j)] a dielectric substrate FR-4. The cross-check analysis proves the relevance of our formulation even in the case where the Babinet's duality is not rigorous, namely, in the presence of the dielectric substrate [Figs. 6(h)-6(n)]. The surface-wave spectra shown in Fig. 6 are in reasonable agreement, both for the semianalytical and numerical results. However, one can see that the characteristic frequencies in the numerical simulation are slightly lower than the ones obtained by the 

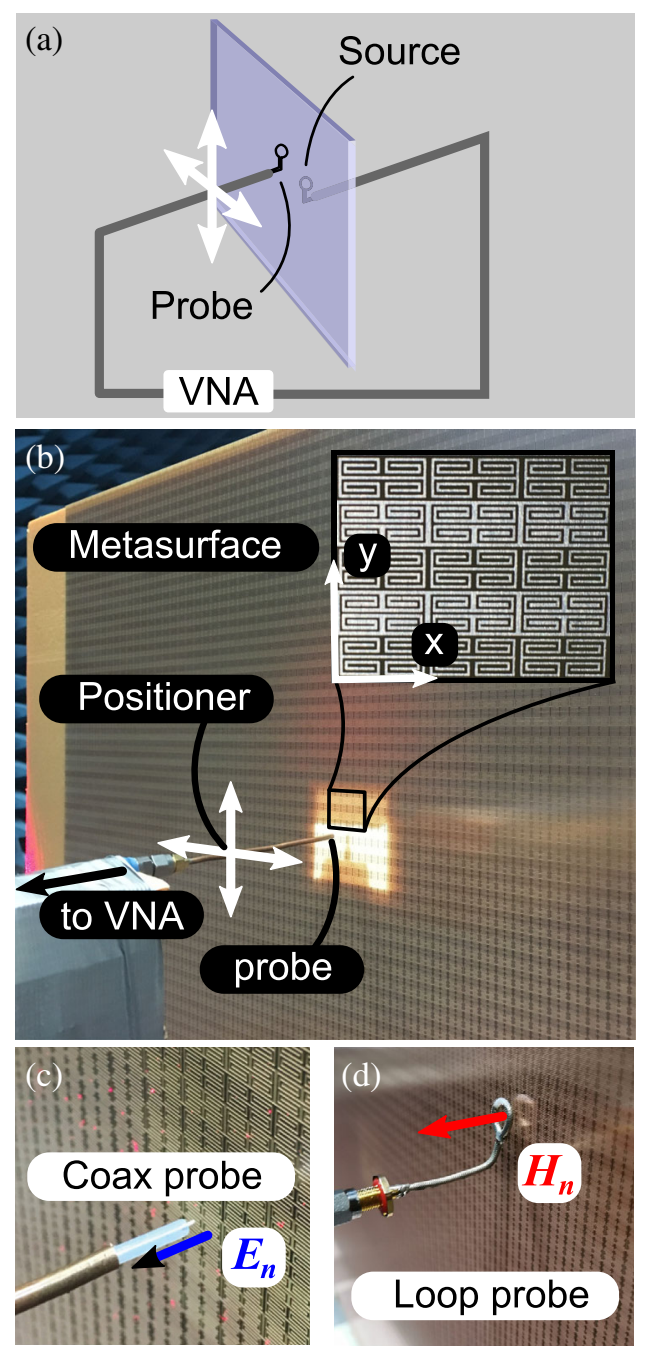

FIG. 7. (a) Scheme and (b) photo of the field measurement setup, and (c) electric and (d) magnetic field probes.

semianalytical process, which is probably a consequence of the nonlocality [61-63].

\section{B. Measurements}

The experimental sample was produced using a printedcircuit-board method on substrate FR-4 with the relative permittivity $\varepsilon=3.9$ and loss tangent $\tan (\delta)=0.02$. The full-size sample has dimension $69 \times 55$ unit cells or $483 \times 385 \mathrm{~mm}^{2}$. The manufactured sample was fixed using a foam substrate and was placed between a source antenna and a probe for near-field measurements in an anechoic chamber [Figs. 7(a) and 7(b)]. The feeding probe during the measurements was fixed and placed in the middle of the structure at a distance of $5 \mathrm{~mm}$ from the sample. At the same time, a scanning probe was moved by a near-field scanner across the plane parallel to the metasurface on the opposite side of the structure such that the gap between the probe and sample was equal to $10 \mathrm{~mm}$. The probe was connected to a vector network analyzer (VNA) Agilent E8362C.
Two complex field maps have been obtained by two independent measurements for quasi-TE and quasi-TM surface waves, respectively. For the measurement of the quasi-TM surface wave, electric monopoles made of openended coaxial cables [Fig. 7(c)] were used both as a source and as a probe to measure the normal component of the electric field. For the quasi-TE surface-wave measurement, Faraday loops $[64,65]$ were manufactured from coaxial cables [Fig. 7(d)] and similarly used as transmitting and receiving probes to measure the normal component of the magnetic field. After the field maps were obtained, they were converted to the isofrequency contours using space Fourier transformation, as was proposed in Refs. $[53,66]$. In our work, we have used fast Fourier transformation with zero padding and a Hamming window to increase the resolution of the isofrequency contours.

The measured and simulated distributions of the normal component of the magnetic field excited by a magnetic loop source (quasi-TE mode) and the reconstructed isofrequency contours are shown in Fig. 8. Similar results for quasi-TM mode fields are shown in Fig. 9.

\section{RESULTS AND DISCUSSION}

\section{A. All-frequency hyperbolicity and hyperbolic plasmons}

The hyperbolicity or the hyperbolic regime of any twodimensional waveguiding systems or metasurfaces arises because of the structure resonances. In the lossless case, the hyperbolic regime could be defined via the condition $\operatorname{Im}\left(Y_{x}\right) \operatorname{Im}\left(Y_{y}\right)<0$ within the effective surface admittance approach. The frequency range corresponding to this condition is strictly limited by the resonant frequencies of the surface admittance tensor components [Fig. 1(a)], which has been shown for various two-dimensional systems in different frequency ranges, including natural materials (e.g., black phosphorus [30,67-69], hexagonal boron nitride [70-73], van der Waals crystals [74,75]) as well as periodic structures composed of graphene strips $[28,68,71]$, gold nanodisks $[63,68,76]$, microstrip transmission lines [77,78], etc. The hyperbolicity can be expected only for the frequencies that are either lower or higher than the resonant frequency. Note that the hyperbolic regime of ultra-anisotropic structures (e.g., silver gratings [31] or split-ring resonators [79] in optical and microwave ranges, respectively) can be broad enough, as shown in Fig. 1(b), but it still remains frequency limited.

The intriguing consequence of Eqs. (4) and (1) is that the hyperbolicity condition $\operatorname{Im}\left(Y_{x}\right) \operatorname{Im}\left(Y_{y}\right)<0$ is met at any frequency [Fig. 1(e) and 1(f)]. The explanation of this physical mechanism lies in the coincidence of the zeros of one surface admittance tensor component, with the poles of the other one related to the mutually orthogonal direction, and vice versa. This pole-zero matching property is guaranteed by the Babinet duality principle (1). Therefore, a 


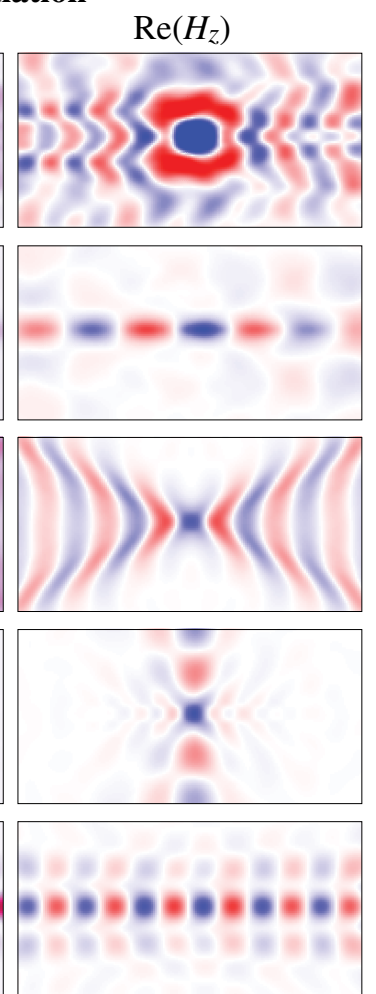
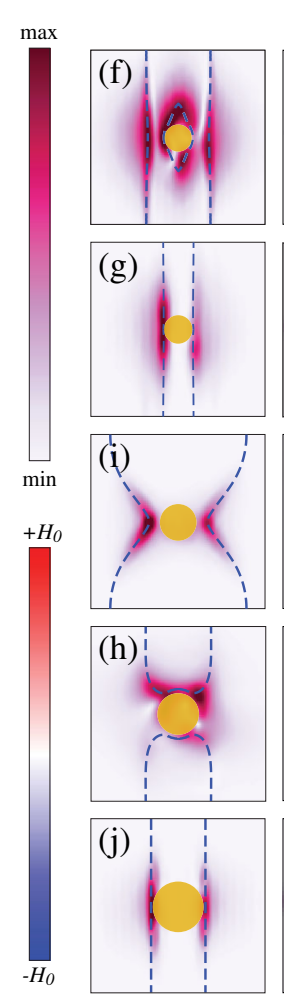
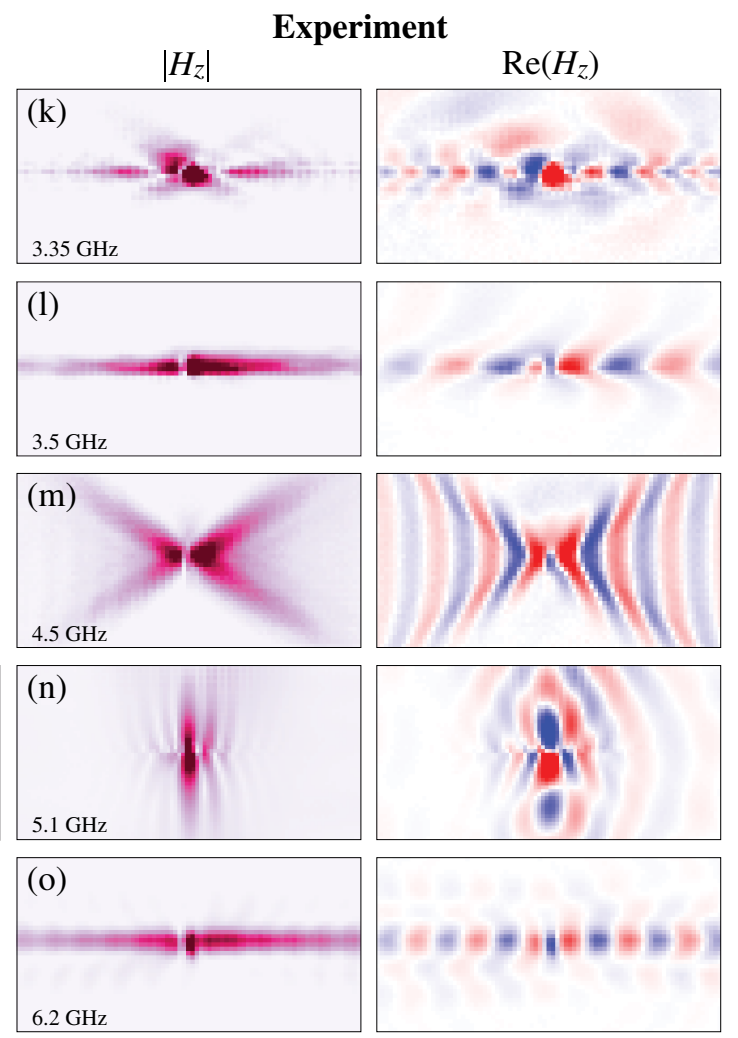

FIG. 8. Spatial distribution of the absolute value (first and fourth columns) and real part (second and fifth columns) of the normal magnetic field component of quasi-TE surface waves calculated numerically (a)-(e) and measured (k)-(o). The scanning area of the structure consists of $48 \times 24$ unit cells $\left(336 \times 168 \mathrm{~mm}^{2}\right)$. The central column (f)-(j) corresponds to the isofrequency contours within the first Brillouin zone restored from the measurements (color maps) and calculated numerically with the CST eigenmode solver (blue dashed lines).

$\left|E_{z}\right|$
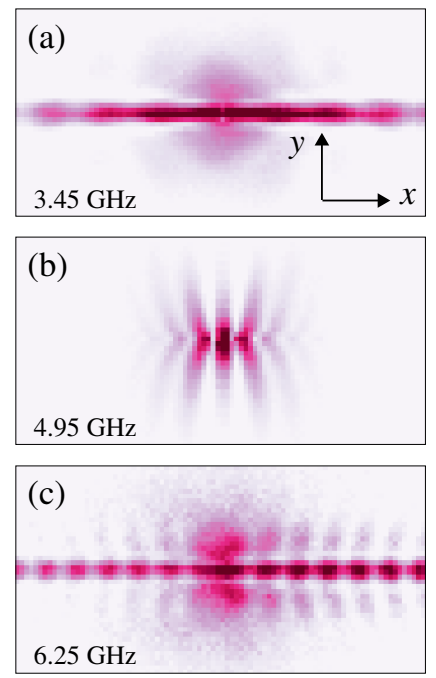

$\operatorname{Re}\left(E_{z}\right)$
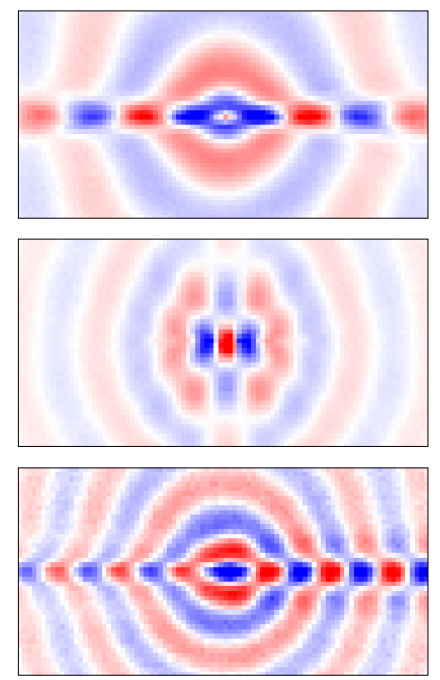

FIG. 9. Spatial distribution of the absolute value (first column) and real part (second column) of the measured normal electric field component for the canalized quasi-TM surface waves. The scanning area of the structure consists of $48 \times 24$ unit cells $\left(336 \times 168 \mathrm{~mm}^{2}\right)$.

self-complementary metasurface appears as a genuine hyperbolic metasurface in the resonant case [Fig. 4(a)]. Hence, the surface waves localized at a resonant self-complementary metasurface can be classified as the hyperbolic plasmonpolaritons $[27,28,30]$.

In contrast to the conventional, relatively narrow-band, hyperbolic operational regime of two-dimensional resonant systems caused by the resonances splitting [Fig. 1(a)], the all-frequency hyperbolicity of a self-complementary metasurface arising from Eq. (1) is a unique feature, and it possesses a high-value practical potential. This result is in full agreement with the IFCs calculated analytically [Figs. 4(e), 4(f), 4(h)] and numerically and reconstructed experimentally [Fig. 8(i)]. Finally, the simulated [Fig. 8(c)] and experimental [Fig. 8(m)] field patterns are characterized by hyperbolic wavefronts. Strictly speaking, the hyperbolicity is supported at any frequency where the Babinet duality principle (1) is fulfilled. One should take into account that, for real structures and materials, this frequency range is always finite, but still, the phenomenon could be extremely broadband, significantly outperforming any other microwave analogues of hyperbolic sheets.

Usually, hyperbolic metasurfaces strongly depend on the geometrical arrangement of the material constituents. 
The slight scaling of the unit-cell size spectrally shifts the resonances and, as a consequence, the frequency range between the resonances corresponding to the hyperbolic regime. Thus, even minor modifications, deformations, and external effects can destroy the hyperbolic regime of a metasurface at a given frequency. This sharp dependence of the hyperbolic properties on the meta-atom scaling and deformation can be denoted as extrinsic hyperbolicity. The opposite situation takes place for natural 2D materials, such as black phosphorus, hexagonal boron nitride, and van der Waals materials, because their hyperbolic properties do not depend on the structure size. In this sense, natural 2D materials possess intrinsic hyperbolicity. Nevertheless, in both cases, the hyperbolic regime is frequency limited. The resonant self-complementary metasurface exhibits both kinds of hyperbolicity. First, it is still an artificial structure, so the scaling spectrally shifts the resonances. At the same time, the resonance shifting for a self-complementary metasurface does not break the hyperbolic regime due to all-frequency hyperbolicity, and for instance, hyperbolic plasmons still exist, in contrast to the purely extrinsic hyperbolicity case. Therefore, we conclude that hyperbolicity of a resonant self-complementary metasurface can be classified as an all-frequency extrinsic, which is in sharp contrast to both conventional hyperbolic metasurfaces and natural hyperbolic materials.

Nevertheless, one should take into account that the hyperbolicity condition $\left[\operatorname{Im}\left(Y_{x}\right) \operatorname{Im}\left(Y_{y}\right)\right]<0$ does not necessarily imply hyperbola-like isofrequency contours of surface waves beyond the effective local approximation. For real implementation, the dispersion of surface waves can be significantly modified, while hyperbola-like IFCs can even be removed, owing to the nonlocal contribution [61-63].

\section{B. Extreme dual-directional canalization}

The necessary conditions for the conventional canalization are the strong anisotropy and near-zero admittance (conductivity) regime $[6,27,37]$. We denote extreme canalization of surface waves as their collinear propagation with ideally flat phase fronts and collimated energy transport like those in Figs. 8 and 9. The conditions $Y_{y} / Y_{x} \rightarrow \infty$ and $Y_{x} / Y_{y} \rightarrow \infty$ lead to the canalization along [Fig. 4(i)] and orthogonal to [Figs. 4(g) and 4(j)] the strips, respectively. One can see that for a self-complementary metasurface, this phenomenon is achieved in the vicinity of $Y_{x}$ and $Y_{y}$ resonances and can be found from the dispersion diagram as the surface plasmon resonances.

To achieve extreme canalization, one has to fulfill both necessary $\left[\left|\operatorname{Im}\left(Y_{x}\right)\right| \ll 1\right]$ and sufficient $\left[\left|\operatorname{Im}\left(Y_{y}\right)\right| \gg 1\right]$ conditions simultaneously in order to achieve the canalization along the $y$ direction $\left[\operatorname{Im}\left(Y_{y}\right) / \operatorname{Im}\left(Y_{x}\right) \rightarrow \infty\right]$ for the lossless case. It is important to note that for the conventional anisotropic structures, the pole $\left[\left|\operatorname{Im}\left(Y_{x}\right)\right| \gg 1\right]$ or zero $\left[\left|\operatorname{Im}\left(Y_{x}\right)\right| \ll 1\right]$ of one surface admittance tensor component (for instance, $Y_{x}$ ) is necessary but not a sufficient condition for the canalization. For general anisotropic metasurfaces, the other surface admittance tensor component $\left(Y_{y}\right)$ is not generally connected to the first one $\left(Y_{x}\right)$.

Another situation takes place for the self-complementary metasurface, whereas the resonant or near-zero behavior of $Y_{x}$ automatically results in the opposite behavior of $Y_{y}$ according to the Babinet duality relation (1). For instance, $Y_{x} \approx 0$ leads to $Y_{y}=4 Y_{0}^{2} / Y_{x} \rightarrow \infty$, so one can observe the extreme vertical canalization under the condition $\left|Y_{y} / Y_{x}\right| \rightarrow \infty$. Thus, any resonance of the selfcomplementary metasurface results in the extreme canalization of surface waves. This result is in sharp contrast to the conventional anisotropic metasurface, whereas the engineering of the canalization phenomenon is much more complicated.

This pole-zero matching inherent to self-complementary metasurfaces results in the extreme canalization and exhibits completely divergenceless propagation with the corresponding, perfectly flat, isofrequency contour. One can compare the flatness of IFCs for the conventional twodimensional anisotropic system [Fig. 1(c)] and the self-complementary metasurface [Fig. 1(g)]. This fundamentally protected regime of extreme canalization at the resonances of the self-complementary metasurface is of high importance from a practical point of view for interferometry [80] and any area needed to route surface waves with specially engineered metasurfaces $[81,82]$.

One can see from the experimental results in Fig. 9 that an effective canalization of quasi-TM surface waves seems to occur only along the strips, while in the practical case, there is no strong localization of the field. The reason for this is related to the shifts of eigenvalue resonant frequencies caused by the nonperfect duality of the measured structure. Indeed, the condition of perfect zero-pole cancellation of the product between two admittance eigenvalues is violated for propagation orthogonal to the strips. In order to obtain an expected extreme canalization in this case, one should adjust the slot dimensions to compensate for the dielectric substrate presence [19]. Besides, the vertical IFC is not purely flat because of the nonlocal interaction between the meta-atoms [Fig. 8(h)]. The analogous effect has previously been observed for waves propagating in a double, mutually orthogonal, wire medium [83]. In this case, the isofrequency contours of a nonlocal practical structure are rather close to two orthogonal flat contours (four straight lines) but transform to the four near-corner hyperbolic lines and a central closed contour because of the capacitive coupling between orthogonal wires.

The promising feature of the resonant self-complementary metasurface (even under weak violation of Babinet's duality principle because of the presence of the dielectric substrate) is the possibility to switch between two orthogonal canalization directions by a small frequency shift. This effect is possible 
because of the existence of at least two consequent resonances of the metasurface. One of the eigenvalues has a zero at a characteristic frequency and a pole at a nearby frequency. The Babinet's principle guarantees that the reverse situation occurs for other eigenvalues related to the orthogonal polarization. We demonstrate, both numerically [Figs. 8(b)-8(e)] and experimentally, the sharp switching between two orthogonal plasmon canalization directions in a narrow operational frequency range. Namely, we measured canalization (i) along the strips at $3.5 \mathrm{GHz}$ [Fig. 8(1)] and 3.45 [Fig. 9(a)], (ii) across the strips at $5.1 \mathrm{GHz}$ [Fig. 8(n)] and 4.95 [Fig. 9(b)], and again (iii) along the strips at $6.2 \mathrm{GHz}$ [Fig. 8(o)] and 6.25 [Fig. 9(c)] for TE (Fig. 8) and TM (Fig. 9) polarization, respectively. This result clearly demonstrates the canalization direction switching (without reflecting mirrors) by applying a minor frequency shift.

\section{All-frequency TE-TM polarization degeneracy}

According to Eq. (10), the dispersions of two modes with orthogonal polarizations are identical for the surface waves on self-complementary metasurface propagation along the main axes directions [Fig. 1(h)]. This feature is inherent to the bulk waves in any isotropic medium where the spectrum is doubly degenerate with respect to polarization. However, in general, for surface waves, the polarization degeneracy phenomenon of eigenmodes is achieved accidentally only at discrete frequency points [Fig. 1(d)]. It has also been shown that the appropriate engineering of an all-dielectric metasurface can lead to the broadband TE-TM degeneracy due to the overlapping of the effective electric and magnetic polarizabilities [84]. One should notice that Eq. (10) uncovers the all-frequency TE-TM degenerate surface waves propagating along the same single-layer two-dimensional structure in four different directions.

In our simple analytical model, the TE-TM polarization degeneracy of the surface waves is fulfilled for the principal directions of the self-complementary metasurface for any frequency [Figs. 4(b) and 4(c)]. Indeed, this result is in good agreement with surface waves on mutually inverted capacitive and inductive metasurfaces [24,25] and topological guided modes between two complementary metasurfaces $[85,86]$. However, in contrast to the previously reported results, anisotropic self-complementary metasurfaces allow frequency-independent polarization degeneracy achievable with any shape of unit cells. In contrast to the topological edge states propagating solely along the edge between two contacting half-surfaces, the considered canalized polarization-degenerate surface waves can be excited by corresponding point sources located at an arbitrary point near the surface. Moreover, several sources can independently launch canalized surface waves with the same or different polarizations, which will then be canalized along parallel, straight paths (see Fig. 2).
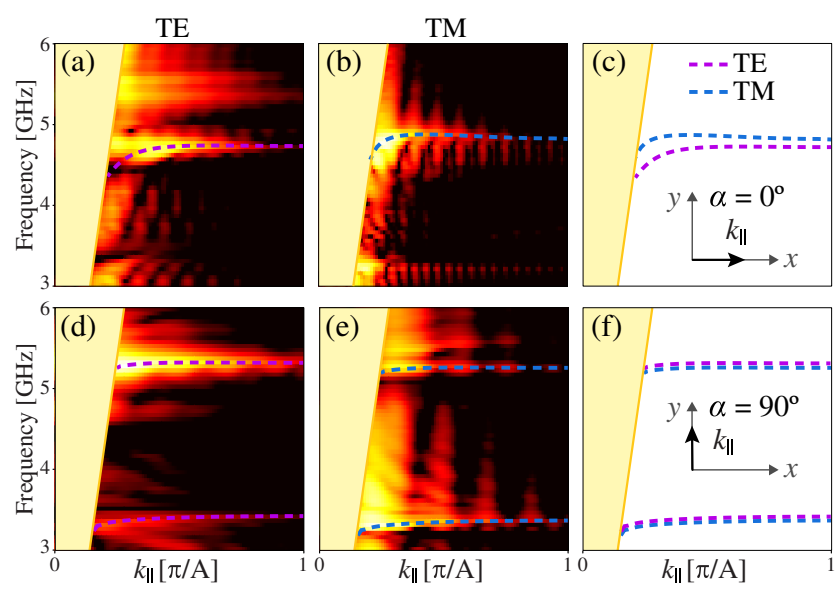

FIG. 10. Reconstructed dispersion of surface waves excited by magnetic (a,d) and electric (b,e) sources propagating along $(a, b)$ and across $(\mathrm{d}, \mathrm{e})$ the strips. We show the comparison of the reconstructed dispersion for TE and TM surface plasmons along (c) and across (f) the strips.

Furthermore, the numerical results, shown in Figs. 6(h)$6(\mathrm{k})$, confirm the polarization degeneracy for the sample, shown in Fig. 5. Besides, we demonstrate a similar dispersion of TE and TM surface modes along and across the strips extracted from the measurements by using the recovered IFCs (Fig. 10), even for a sample on a substrate.

The demonstrated effect of broadband polarization degeneracy of surface waves is especially pronounced in the experiment for the canalization regimes. The frequencies corresponding to the canalization regime for quasi-TE [Figs. 8(1), 8(n), and 8(o)] and quasi-TM (Fig. 9) modes are almost the same. Therefore, we state that our resonant selfcomplementary metasurface in the vicinity of its resonances can support highly directional propagation of two orthogonally polarized surface waves with nearly the same phase velocity, which means that the canalized plasmon can be transmitted in the direction parallel to one of the main axes, keeping the initial polarization of its source (e.g., circular, linear, or elliptical polarization). Moreover, the effect of canalization allows for parallel routing of surface waves excited by multiple sources located near the same metasurface. This operational principle can enhance the sensing of chiral molecules sensitive to polarization [7] and expand the element base and functionality of planar photonic devices, including flat polarizers and multiplexers of surface waves, while in the microwave range, it can be directly applied to engineer the planar, holographic, leaky-wave antennas based on the modulated surface impedance with the capability to operate with arbitrary polarization in a multichannel mode.

\section{CONCLUSIONS}

To conclude, we have studied, both theoretically and experimentally, the surface waves localized at a resonant 
self-complementary metasurface at microwaves. We have developed a consistent analytical model based on the effective surface admittance tensor approach. We have theoretically, numerically, and experimentally demonstrated the all-frequency intrinsic hyperbolicity, canalization in frequency-switchable direction with ultimately flat wavefronts (extreme canalization), and broadband TE-TM polarization degeneracy of surface waves localized at a selfcomplementary metasurface. Importantly, we have shown the simultaneous canalization and TE-TM degeneracy of surface waves in the vicinity of the self-complementary metasurface resonances. Thus, the emitter energy can be transferred by virtue of highly directive surface waves, keeping the same polarization.

The resonant self-complementary metasurfaces could be easily scaled up to the terahertz region, where the metals can still be approximately represented as perfect electric conductors. For higher frequencies, in plasmonic frequency windows, the metals exhibit strong dispersion and high absorption losses, significantly limiting the Babinet inversion principle to the narrow frequency range. Particularly, plasmonic metasurfaces can fulfill the Babinet principle in the vicinity of the resonance $[87,88]$, opening up the possibility of simultaneous canalization and polarization degeneracy of surface waves. Another approach to implement the self-complementary metasurfaces in the visible can include the all-dielectric materials (such as silicon and oxides) exhibiting a weak dispersion and negligibly small absorption in the optical and near-infrared ranges. The alldielectric metasurfaces can exhibit the sharp collective resonant response caused by Mie resonances of the intercoupled particles [89]. The latter can be tailored in shape and size to enhance TE or TM local resonance, thus having an equivalent complementary homogenized behavior. Finally, the Babinet principle could be fulfilled in a broadband frequency range for active and tunable metasurfaces, whereas the external factors (voltage, magnetic field, temperature, mechanical rotation, loaded capacitors) can compensate the strong dispersion appropriately [90-93].

In the microwave range, surface waves localized at selfcomplementary metasurfaces represent a low-loss, simple and cheap method for guiding localized electromagnetic signals. Furthermore, the broadband TE-TM polarization degeneracy can be exploited, along with the extreme canalization, to design dual-polarized leaky-wave antennas with azimuthal beam scanning capability. The results obtained reveal that the self-complementary metasurfaces provide the platform for a number of applications, including polarization control and routing over optical and radio-frequency signals, on-chip devices, planar networks, photonic components, antennas, and sensors.

\section{ACKNOWLEDGMENTS}

The analytical and numerical results have been supported by the Russian Foundation for Basic Research
(Grant No. 20-02-00636). The fabrication and experimental study have been supported by the Russian Science Foundation (Grant No. 21-79-30038). O. Y. acknowledges support from the Foundation for the Advancement of Theoretical Physics and Mathematics "BASIS." The authors thank an anonymous referee for helping them highlight the novelty and uniqueness of the results presented, as well as Professors Andrey Bogdanov and Pavel Belov from ITMO University for fruitful discussions.

[1] C. L. Holloway, E. F. Kuester, J. A. Gordon, J. O'Hara, J. Booth, and D. R. Smith, An Overview of the Theory and Applications of Metasurfaces: The Two-Dimensional Equivalents of Metamaterials, IEEE Antennas Propag. Mag. 54, 10 (2012).

[2] A. V. Kildishev, A. Boltasseva, and V. M. Shalaev, Planar Photonics with Metasurfaces, Science 339, 1232009 (2013).

[3] N. Yu and F. Capasso, Flat Optics with Designer Metasurfaces, Nat. Mater. 13, 139 (2014).

[4] S. B. Glybovski, S. A. Tretyakov, P. A. Belov, Y. S. Kivshar, and C. R. Simovski, Phys. Rep. 634, 1 (2016).

[5] H.-T. Chen, A. J. Taylor, and N. Yu, A Review of Metasurfaces: Physics and Applications, Rep. Prog. Phys. 79, 076401 (2016).

[6] J. Gomez-Diaz and A. Alù, Flatland Optics with Hyperbolic Metasurfaces, ACS Photonics 3, 2211 (2016).

[7] S. Droulias and L. Bougas, Surface Plasmon Platform for Angle-Resolved Chiral Sensing, ACS Photonics 6, 1485 (2019).

[8] S. Maci, G. Minatti, M. Casaletti, and M. Bosiljevac, Metasurfing: Addressing Waves on Impenetrable Metasurfaces, IEEE Antennas Wireless Propag. Lett. 10, 1499 (2011).

[9] B. H. Fong, J. S. Colburn, J. J. Ottusch, J. L. Visher, and D. F. Sievenpiper, Scalar and Tensor Holographic Artificial Impedance Surfaces, IEEE Trans. Antennas Propag. 58, 3212 (2010).

[10] G. Minatti, F. Caminita, M. Casaletti, and S. Maci, Spiral Leaky-Wave Antennas Based on Modulated Surface Impedance, IEEE Trans. Antennas Propag. 59, 4436 (2011).

[11] G. Minatti, S. Maci, P. De Vita, A. Freni, and M. Sabbadini, A Circularly-Polarized Isoflux Antenna Based on Anisotropic Metasurface, IEEE Trans. Antennas Propag. 60, 4998 (2012).

[12] C. Pfeiffer and A. Grbic, A Printed, Broadband Luneburg Lens Antenna, IEEE Trans. Antennas Propag. 58, 3055 (2010).

[13] M. Bosiljevac, M. Casaletti, F. Caminita, Z. Sipus, and S. Maci, Non-Uniform Metasurface Luneburg Lens Antenna Design, IEEE Trans. Antennas Propag. 60, 4065 (2012).

[14] J. A. Dockrey, M. J. Lockyear, S. J. Berry, S. A. R. Horsley, J. R. Sambles, and A. P. Hibbins, Thin Metamaterial Luneburg Lens for Surface Waves, Phys. Rev. B 87, 125137 (2013).

[15] X. Wan, X. Shen, Y. Luo, and T. J. Cui, Planar Bifunctional Luneburg-Fisheye Lens Made of an Anisotropic Metasurface, Laser Photonics Rev. 8, 757 (2014).

[16] J. Ortiz, J. Baena, V. Losada, F. Medina, R. Marques, and J.A. Quijano, Self-Complementary Metasurface for 
Designing Narrow Band Pass/Stop Filters, IEEE Microwave Wireless Compon. Lett. 23, 291 (2013).

[17] Y. Urade, Y. Nakata, T. Nakanishi, and M. Kitano, Broadband and Energy-Concentrating Terahertz Coherent Perfect Absorber Based on a Self-Complementary Metasurface, Opt. Lett. 41, 4472 (2016).

[18] J. D. Baena, J.P. del Risco, A. P. Slobozhanyuk, S. B. Glybovski, and P. A. Belov, Self-Complementary Metasurfaces for Linear-to-Circular Polarization Conversion, Phys. Rev. B 92, 245413 (2015).

[19] J. D. Baena, S. B. Glybovski, J. P. del Risco, A. P. Slobozhanyuk, and P. A. Belov, Broadband and Thin Linear-to-Circular Polarizers Based on Self-Complementary Zigzag Metasurfaces, IEEE Trans. Antennas Propag. 65, 4124 (2017).

[20] A. Sayanskiy, S. A. Kuznetsov, D. S. Tanygina, J. P. Del Risco, S. Glybovski, and J. D. Baena, FrequencyControllable Polarization Rotation of $\mathrm{THz}$ Waves with an SCMS, IEEE Trans. Antennas Propag. 68, 1491 (2020).

[21] R. Compton, J. Macfarlane, L. Whitbourn, M. Blanco, and R. McPhedran, Babinet's Principle Applied to Ideal BeamSplitters for Submillimetre Waves, Opt. Acta 31, 515 (1984).

[22] Y. Nakata, Y. Urade, T. Nakanishi, and M. Kitano, PlaneWave Scattering by Self-Complementary Metasurfaces in Terms of Electromagnetic Duality and Babinet's Principle, Phys. Rev. B 88, 205138 (2013).

[23] Y. Urade, Y. Nakata, T. Nakanishi, and M. Kitano, Frequency-Independent Response of Self-Complementary Checkerboard Screens, Phys. Rev. Lett. 114, 237401 (2015).

[24] D. González-Ovejero, E. Martini, and S. Maci, Surface Waves Supported by Metasurfaces with Self-Complementary Geometries, IEEE Trans. Antennas Propag. 63, 250 (2015).

[25] M. Li, S. Xiao, J. Long, and D. F. Sievenpiper, Surface Waveguides Supporting Both TM Mode and TE Mode with the Same Phase Velocity, IEEE Trans. Antennas Propag. 64, 3811 (2016).

[26] H. G. Booker, Slot Aerials and Their Relation to Complementary Wire Aerials (Babinet's Principle), J. Inst. Electr. Eng. IIIA 93, 620 (1946).

[27] O. Y. Yermakov, A. I. Ovcharenko, M. Song, A. A. Bogdanov, I. V. Iorsh, and Y. S. Kivshar, Hybrid Waves Localized at Hyperbolic Metasurfaces, Phys. Rev. B 91, 235423 (2015).

[28] J. S. Gomez-Diaz, M. Tymchenko, and A. Alù, Hyperbolic Plasmons and Topological Transitions over Uniaxial Metasurfaces, Phys. Rev. Lett. 114, 233901 (2015).

[29] M. Mencagli, E. Martini, and S. Maci, Surface Wave Dispersion for Anisotropic Metasurfaces Constituted by Elliptical Patches, IEEE Trans. Antennas Propag. 63, 2992 (2015).

[30] A. Nemilentsau, T. Low, and G. Hanson, Anisotropic 2D Materials for Tunable Hyperbolic Plasmonics, Phys. Rev. Lett. 116, 066804 (2016).

[31] A. A. High, R. C. Devlin, A. Dibos, M. Polking, D. S. Wild, J. Perczel, N. P. de Leon, M. D. Lukin, and H. Park, VisibleFrequency Hyperbolic Metasurface, Nature (London) 522, 192 (2015).

[32] I. Liberal and N. Engheta, Near-Zero Refractive Index Photonics, Nat. Photonics 11, 149 (2017).
[33] O. Kotov and Y. E. Lozovik, Hyperbolic Hybrid Waves and Optical Topological Transitions in Few-Layer Anisotropic Metasurfaces, Phys. Rev. B 100, 165424 (2019).

[34] G. Hu, A. Krasnok, Y. Mazor, C.-W. Qiu, and A. Alù, Moiré Hyperbolic Metasurfaces, Nano Lett. 20, 3217 (2020).

[35] S. A. H. Gangaraj, G. W. Hanson, M. G. Silveirinha, K. Shastri, M. Antezza, and F. Monticone, Unidirectional and Diffractionless Surface Plasmon Polaritons on ThreeDimensional Nonreciprocal Plasmonic Platforms, Phys. Rev. B 99, 245414 (2019).

[36] A. Nemilentsau, T. Stauber, G. Gómez-Santos, M. Luskin, and T. Low, Switchable and Unidirectional Plasmonic Beacons in Hyperbolic Two-Dimensional Materials, Phys. Rev. B 99, 201405(R) (2019).

[37] D. Correas-Serrano, A. Alù, and J. S. Gomez-Diaz, Plasmon Canalization and Tunneling over Anisotropic Metasurfaces, Phys. Rev. B 96, 075436 (2017).

[38] See Supplemental Material at http://link.aps.org/ supplemental/10.1103/PhysRevX.11.031038 for the analytical comparison of the effective surface admittance tensors of hyperbolic and self-complementary metasurfaces, and the practical guideline for the self-complementary metasurfaces designing.

[39] It is also known as collimation, guiding, channeling, and tunneling.

[40] H. Kosaka, T. Kawashima, A. Tomita, M. Notomi, T. Tamamura, T. Sato, and S. Kawakami, Self-Collimating Phenomena in Photonic Crystals, Appl. Phys. Lett. 74, 1212 (1999).

[41] J. Witzens, M. Loncar, and A. Scherer, Self-Collimation in Planar Photonic Crystals, IEEE J. Sel. Top. Quantum Electron. 8, 1246 (2002).

[42] P. A. Belov, C. R. Simovski, and P. Ikonen, Canalization of Subwavelength Images by Electromagnetic Crystals, Phys. Rev. B 71, 193105 (2005).

[43] A. Salandrino and N. Engheta, Far-Field Subdiffraction Optical Microscopy Using Metamaterial Crystals: Theory and Simulations, Phys. Rev. B 74, 075103 (2006).

[44] N.-H. Shen, P. Zhang, T. Koschny, and C. M. Soukoulis, Metamaterial-Based Lossy Anisotropic Epsilon-Near-Zero Medium for Energy Collimation, Phys. Rev. B 93, 245118 (2016).

[45] P. Ikonen, P. Belov, C. Simovski, and S. Maslovski, Experimental Demonstration of Subwavelength Field Channeling at Microwave Frequencies Using a Capacitively Loaded Wire Medium, Phys. Rev. B 73, 073102 (2006).

[46] P. A. Belov and Y. Hao, Subwavelength Imaging at Optical Frequencies Using a Transmission Device Formed by a Periodic Layered Metal-Dielectric Structure Operating in the Canalization Regime, Phys. Rev. B 73, 113110 (2006).

[47] M. G. Silveirinha, P. A. Belov, and C. R. Simovski, Subwavelength Imaging at Infrared Frequencies Using an Array of Metallic Nanorods, Phys. Rev. B 75, 035108 (2007).

[48] B. Stein, E. Devaux, C. Genet, and T. Ebbesen, SelfCollimation of Surface Plasmon Beams, Opt. Lett. 37, 1916 (2012).

[49] O. Y. Yermakov, A. A. Hurshkainen, D. A. Dobrykh, P. V. Kapitanova, I. V. Iorsh, S. B. Glybovski, and 
A. A. Bogdanov, Experimental Observation of Hybrid TETM Polarized Surface Waves Supported by a Hyperbolic Metasurface, Phys. Rev. B 98, 195404 (2018).

[50] O. Y. Yermakov, A. I. Ovcharenko, A. A. Bogdanov, I. V. Iorsh, K. Y. Bliokh, and Y. S. Kivshar, Spin Control of Light with Hyperbolic Metasurfaces, Phys. Rev. B 94, 075446 (2016).

[51] P. V. Kapitanova, P. Ginzburg, F. J. Rodríguez-Fortuño, D. S. Filonov, P. M. Voroshilov, P. A. Belov, A. N. Poddubny, Y.S. Kivshar, G. A. Wurtz, and A. V. Zayats, Photonic Spin Hall Effect in Hyperbolic Metamaterials for Polarization-Controlled Routing of Subwavelength Modes, Nat. Commun. 5, 3226 (2014).

[52] A. Hayat, J. B. Mueller, and F. Capasso, Lateral ChiralitySorting Optical Forces, Proc. Natl. Acad. Sci. U.S.A. 112, 13190 (2015).

[53] Y. Yang, L. Jing, L. Shen, Z. Wang, B. Zheng, H. Wang, E. Li, N.-H. Shen, T. Koschny, C. M. Soukoulis, and H. Chen, Hyperbolic Spoof Plasmonic Metasurfaces, NPG Asia Mater. 9, e428 (2017).

[54] Y. Mazor and A. Alù, Routing Optical Spin and Pseudospin with Metasurfaces, Phys. Rev. Applied 14, 014029 (2020).

[55] F. J. Rodríguez-Fortuño, G. Marino, P. Ginzburg, D. OConnor, A. Martínez, G. A. Wurtz, and A. V. Zayats, Near-Field Interference for the Unidirectional Excitation of Electromagnetic Guided Modes, Science 340, 328 (2013).

[56] J. Lin, J. B. Mueller, Q. Wang, G. Yuan, N. Antoniou, X.-C. Yuan, and F. Capasso, Polarization-Controlled Tunable Directional Coupling of Surface Plasmon Polaritons, Science 340, 331 (2013).

[57] D. OConnor, P. Ginzburg, F. J. Rodríguez-Fortuño, G. A. Wurtz, and A. V. Zayats, Spin-Orbit Coupling in Surface Plasmon Scattering by Nanostructures, Nat. Commun. 5, 5327 (2014).

[58] I. Sinev, F. Komissarenko, I. Iorsh, D. Permyakov, A. Samusev, and A. Bogdanov, Steering of Guided Light with Dielectric Nanoantennas, ACS Photonics 7, 680 (2020).

[59] C. Meng, S. Tang, F. Ding, and S. I. Bozhevolnyi, Optical Gap-Surface Plasmon Metasurfaces for Spin-Controlled Surface Plasmon Excitation and Anomalous Beam Steering, ACS Photonics 7, 1849 (2020).

[60] D. M. Pozar, Microwave Engineering (John Wiley \& Sons, New York, 2009).

[61] M. A. Gorlach and P. A. Belov, Effect of Spatial Dispersion on the Topological Transition in Metamaterials, Phys. Rev. B 90, 115136 (2014).

[62] D. Correas-Serrano, J. Gomez-Diaz, M. Tymchenko, and A. Alù, Nonlocal Response of Hyperbolic Metasurfaces, Opt. Express 23, 29434 (2015).

[63] O. Y. Yermakov, D. V. Permyakov, F. V. Porubaev, P. A. Dmitriev, A. K. Samusev, I. V. Iorsh, R. Malureanu, A. V. Lavrinenko, and A. A. Bogdanov, Effective Surface Conductivity of Optical Hyperbolic Metasurfaces: From FarField Characterization to Surface Wave Analysis, Sci. Rep. 8, 14135 (2018).

[64] J. D. Jackson, Classical Electrodynamics, 3rd ed. (Wiley, New York, NY, 1999).

[65] J. B. Pendry, A. J. Holden, D. J. Robbins, and W. J. Stewart, Magnetism from Conductors and Enhanced Nonlinear Phenomena, IEEE Trans. Microwave Theory Tech. 47, 2075 (1999).
[66] J. A. Dockrey, S. A. R. Horsley, I. R. Hooper, J. R. Sambles, and A.P. Hibbins, Direct Observation of Negative-Index Microwave Surface Waves, Sci. Rep. 6, 22018 (2016).

[67] D. Correas-Serrano, J. Gomez-Diaz, A. A. Melcon, and A. Alù, Black Phosphorus Plasmonics: Anisotropic Elliptical Propagation and Nonlocality-Induced Canalization, J. Opt. 18, 104006 (2016).

[68] O. Kotov and Y. E. Lozovik, Enhanced Optical Activity in Hyperbolic Metasurfaces, Phys. Rev. B 96, 235403 (2017).

[69] E. Van Veen, A. Nemilentsau, A. Kumar, R. Roldán, M. I. Katsnelson, T. Low, and S. Yuan, Tuning Two-Dimensional Hyperbolic Plasmons in Black Phosphorus, Phys. Rev. Applied 12, 014011 (2019).

[70] S. Dai, Q. Ma, T. Andersen, A. Mcleod, Z. Fei, M. Liu, M. Wagner, K. Watanabe, T. Taniguchi, M. Thiemens, F. Keilmann, P. Jarillo-Herrero, M. Fogler, and D. Basov, Subdiffractional Focusing and Guiding of Polaritonic Rays in a Natural Hyperbolic Material, Nat. Commun. 6, 6963 (2015).

[71] T. Low, A. Chaves, J. D. Caldwell, A. Kumar, N. X. Fang, P. Avouris, T. F. Heinz, F. Guinea, L. Martin-Moreno, and F. Koppens, Polaritons in Layered Two-Dimensional Materials, Nat. Mater. 16, 182 (2017).

[72] A. A. Govyadinov, A. Konečná, A. Chuvilin, S. Vélez, I. Dolado, A. Y. Nikitin, S. Lopatin, F. Casanova, L. E. Hueso, J. Aizpurua, and R. Hillenbrand, Probing Low-Energy Hyperbolic Polaritons in van der Waals Crystals with an Electron Microscope, Nat. Commun. 8, 95 (2017).

[73] P. Li, I. Dolado, F. J. Alfaro-Mozaz, F. Casanova, L. E. Hueso, S. Liu, J.H. Edgar, A. Y. Nikitin, S. Vélez, and R. Hillenbrand, Infrared Hyperbolic Metasurface Based on Nanostructured van der Waals Materials, Science 359, 892 (2018).

[74] Z. Zheng, N. Xu, S. L. Oscurato, M. Tamagnone, F. Sun, Y. Jiang, Y. Ke, J. Chen, W. Huang, W. L. Wilson, A. Ambrosio, S. Deng, and H. Chen, A Mid-Infrared Biaxial Hyperbolic van der Waals Crystal, Sci. Adv. 5, eaav8690 (2019).

[75] C. Wang, S. Huang, Q. Xing, Y. Xie, C. Song, F. Wang, and H. Yan, Van der Waals Thin Films of $\mathrm{WTe}_{2}$ for Natural Hyperbolic Plasmonic Surfaces, Nat. Commun. 11, 1158 (2020).

[76] A. Samusev, I. Mukhin, R. Malureanu, O. Takayama, D. V. Permyakov, I. S. Sinev, D. Baranov, O. Yermakov, I. V. Iorsh, A. A. Bogdanov, and A. V. Lavrinenko, Polarization-Resolved Characterization of Plasmon Waves Supported by an Anisotropic Metasurface, Opt. Express 25, 32631 (2017).

[77] A. V. Chshelokova, P. V. Kapitanova, A. N. Poddubny, D. S. Filonov, A. P. Slobozhanyuk, Y. S. Kivshar, and P. A. Belov, Hyperbolic Transmission-Line Metamaterials, J. Appl. Phys. 112, 073116 (2012).

[78] S. H. Sedighy, C. Guclu, S. Campione, M. K. Amirhosseini, and F. Capolino, Wideband Planar Transmission Line Hyperbolic Metamaterial for Subwavelength Focusing and Resolution, IEEE Trans. Microwave Theory Tech. 61, 4110 (2013).

[79] Y. Yang, P. Qin, B. Zheng, L. Shen, H. Wang, Z. Wang, E. Li, R. Singh, and H. Chen, Magnetic Hyperbolic Metasurface: Concept, Design, and Applications, Adv. Sci. 5, 1801495 (2018). 
[80] D. Zhao, J. Zhang, P. Yao, X. Jiang, and X. Chen, Photonic Crystal Mach-Zehnder Interferometer Based on SelfCollimation, Appl. Phys. Lett. 90, 231114 (2007).

[81] S. A. H. Gangaraj, T. Low, A. Nemilentsau, and G. W. Hanson, Directive Surface Plasmons on Tunable TwoDimensional Hyperbolic Metasurfaces and Black Phosphorus: Greens Function and Complex Plane Analysis, IEEE Trans. Antennas Propag. 65, 1174 (2016).

[82] K. Achouri and C. Caloz, Space-Wave Routing via Surface Waves Using a Metasurface System, Sci. Rep. 8, 7549 (2018).

[83] C. Simovski and P. Belov, Low-Frequency Spatial Dispersion in Wire Media, Phys. Rev. E 70, 046616 (2004).

[84] O. Y. Yermakov, A. A. Bogdanov, and A. V. Lavrinenko, Broadband Polarization Degeneracy of Guided Waves in Subwavelength Structured ZnO Pattern, IEEE J. Sel. Top. Quantum Electron. 25, 1 (2019).

[85] D. J. Bisharat and D. F. Sievenpiper, Guiding Waves along an Infinitesimal Line between Impedance Surfaces, Phys. Rev. Lett. 119, 106802 (2017).

[86] D. J. Bisharat and D. F. Sievenpiper, Electromagnetic-Dual Metasurfaces for Topological States along a 1D Interface, Laser Photonics Rev. 13, 1900126 (2019).

[87] T. Zentgraf, T. Meyrath, A. Seidel, S. Kaiser, H. Giessen, C. Rockstuhl, and F. Lederer, Babinets Principle for Optical
Frequency Metamaterials and Nanoantennas, Phys. Rev. B 76, 033407 (2007).

[88] M. Zalkovskij, R. Malureanu, C. Kremers, D. N. Chigrin, A. Novitsky, S. Zhukovsky, P. T. Tang, P. U. Jepsen, and A. V. Lavrinenko, Optically Active Babinet Planar Metamaterial Film for Terahertz Polarization Manipulation, Laser Photonics Rev. 7, 810 (2013).

[89] S. Kruk and Y. Kivshar, Functional Meta-Optics and Manophotonics Governed by Mie Resonances, ACS Photonics 4, 2638 (2017).

[90] Y. Urade, Y. Nakata, K. Okimura, T. Nakanishi, F. Miyamaru, M. W. Takeda, and M. Kitano, Dynamically Babinet-Invertible Metasurface: A Capacitive-Inductive Reconfigurable Filter for Terahertz Waves Using Vanadium-Dioxide MetalInsulator Transition, Opt. Express 24, 4405 (2016).

[91] C. Zou, J. Sautter, F. Setzpfandt, and I. Staude, Resonant Dielectric Metasurfaces: Active Tuning and Nonlinear Effects, J. Phys. D 52, 373002 (2019).

[92] F. Ding, Y. Yang, and S. I. Bozhevolnyi, Dynamic Metasurfaces Using Phase-Change Chalcogenides, Adv. Opt. Mater. 7, 1801709 (2019).

[93] C. R. de Galarreta, I. Sinev, A. M. Alexeev, P. Trofimov, K. Ladutenko, S. G.-C. Carrillo, E. Gemo, A. Baldycheva, J. Bertolotti, and C.D. Wright, Reconfigurable Multilevel Control of Hybrid All-Dielectric Phase-Change Metasurfaces, Optica 7, 476 (2020). 